\title{
Le point sur les expositions accidentelles connues
}

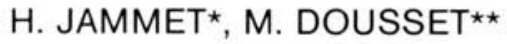 \\ (Manuscrit reçu le 15 octobre 1984)
}

\begin{abstract}
RÉSUMÉ
Cet article fait le point sur les accidents connus qui, depuis 40 ans, ont entraîné, par exposition externe ou interne aux rayonnements ionisants, des décès, des syndromes graves d'irradiation globale, ou des expositions localisées ayant justifié des soins médicaux attentifs et souvent des interventions chirurgicales.

La plupart de ces expositions accidentelles sont survenues au cours de : $1 \%$ travaux sur des dispositifs expérimentaux ; $2 \%$ manipulation et détention de sources ramassées par ignorance ; $3 \%$ utilisation industrielle ou médicale de sources scellées ou non scellées.
\end{abstract}

Elles sont pratiquement inexistantes dans les activités spécifiques des grandes installations du cycle du combustible nucléaire.

\section{ABSTRACT}

A review is made of the external or internal overexposures to ionizing radiation that have occured for $\mathbf{4 0}$ years and have entailed deaths, acute whole-body or partial-body syndromes requiring close medical care and often surgery.

Most overexposures occured during : $1 /$ work on experimental devices ; $2 /$ handling or keeping of sources collected by ignorance ; 3 / industrial or medical uses of sealed or unsealed sources. Practically no overexposures have occured in the activities specific of the large utilities in the nuclear fuel cycle.

II n'est pas, dans l'histoire de l'humanité, de découvertes scientifiques touchant aux sources d'énergie dont les premières applications n'aient entraîné quelques préjudices pour l'homme. Ce n'est qu'avec les progrès de la connaissance et la maîtrise des phénomènes que ces situations regrettables sont devenues plus rares et ont revêtu plus ou moins rapidement un caractère exceptionnel.

Les applications des rayonnements ionisants qui ont suivi la découverte des rayons de Röntgen en 1895 puis celle des substances radioactives avec Becquerel en 1896 et Pierre et Marie Curie en 1898 n'ont pas échappé

* Commissariat à l'énergie atomique, Institut de protection et de sûreté nucléaire, BP 6, 92260 Fontenay-aux-Roses.

* Conseiller scientifique du Service central de protection contre les rayonnements ionisants, 7, rue de la Gruerie, 91190 Gif-sur-Yvette. 
à cette fatalité. Dès 1896, Stevens [10] fut un des premiers à décrire une radiolésion cutanée chez un manipulateur d'appareil à rayons $X$, et Daniel [3] relatait, la même année, la chute des cheveux chez un très jeune enfant longuement exposé aux rayons $X$ pour la recherche d' un projectile intracrânien.

Avec les rayonnements issus des substances radioactives naturelles, les mêmes effets furent observés: Becquerel et Curie [1] furent eux-mêmes victimes de radionécroses de la peau.

La découverte de la radioactivité artificielle (1934), celle de la fission (1939), suivies de leurs applications et, en particulier, du développement de l'énergie nucléaire à des fins militaires et civiles multiplièrent les occasions d'exposition accidentelle ; mais, en contrepartie, les progrès dans la sûreté des installations comme dans les moyens de prévention et de surveillance rendirent assez vite ces situations accidentelles relativement exceptionnelles.

Dans le bref historique qui suit, il a paru opportun de limiter le sujet aux événements accidentels qui se sont produits après 1945 et de ne retenir que ceux où les sujets atteints ont présenté un état justifiant des soins attentifs.

On peut diviser ce travail en deux parties d'inégale importance selon qu'on envisage :

1. Les événements accidentels de grande portée ;

2. Les événements accidentels de portée restreinte.

\section{LES ÉVÉNEMENTS ACCIDENTELS DE GRANDE PORTÉE}

Ce sont des événements très rares. Si on élimine l'accident de Windscale (Grande-Bretagne) des 10 et 11 octobre 1957 (incendie d'un réacteur à uranium naturel et graphite refroidi à l'air) et celui de Three Mile Island (Etats-Unis), le 28 mars 1979, accidents qui n'ont fait aucune victime au sens précisé plus haut, on ne peut citer comme fait certain dans cette catégorie que deux événements :

- les retombées radioactives sur les îles Marshall et sur le bateau de pêche japonais Fukuryumaru le $1^{\text {er }}$ mars $1954[4,5]$;

- la dispersion des grains aimantés d'une source de cobalt 60 au Mexique en décembre 1983.

L'accident qui serait responsable de la dévastation d'une région relativement grande près de Kyshtym (URSS) dans le sud-est de l'Oural au cours de l'hiver 1957-1958 n'est encore qu'une hypothèse [7 - 9].

a) Les fortes retombées radioactives qui ont suivi l'essai dans le Pacifique du premier engin thermonucléaire opérationnel des USA ont principalement atteint, à environ 100 miles de Bikini, 67 personnes sur l'atoll de Rongelap, 19 sur Airlinginae et les 23 hommes de l'équipage du bâteau de pêche japonais. Le passage du nuage et surtout le dépôt de poussières radioactives visibles ont entraîné une exposition externe par rayonnements $\gamma$ (pénétrants) allant de 0,7 à 3,20 grays (70 à 320 rads) et une exposition $\beta$ de l'ordre de une à plusieurs dizaines de grays au niveau des surfaces découvertes du corps (les pieds principalement). En outre, les autochtones, durant leur séjour sur les atolls en attendant leur évacuation, et les pêcheurs au cours de leur voyage de retour au Japon, qui dura treize jours, ont 
inhalé et ingéré des produits de fission, ce qui se traduisit par une importante exposition interne.

Les effets précoces : brûlures de la peau, nausées, vomissements, perturbation de la formule sanguine, etc., se manifestèrent principalement chez les habitants de Rongelap et chez les pêcheurs japonais ; l'un de ces pêcheurs mourut six mois après.

Parmi les effets tardifs qui apparurent entre dix et vingt ans après chez les autochtones des atolls, ce sont les anomalies et les cancers de la glande thyroïde qui furent les plus fréquents, surtout chez ceux qui étaient enfants lors de l'accident et tout spécialement chez les filles.

b) A la fin de l'année 1983 , le conteneur d'une source de cobalt 60 pour appareil de télégammathérapie était vendu, avec la source, à un ferailleur de Ciudad Juarez (Mexique). Après une chute du conteneur, les grains (7200) de cobalt furent disséminés et en partie répandus sur la chaussée et dans le camion avec lequel on transportait la source. Quelques-uns des ouvriers de l'entreprise, cinq semble-t-il, furent sévèrement exposés ; ils reçurent des doses de 1 à 5 Gy (100 à 500 rads) étalées sur plusieurs semaines. Dans la ville et les environs, 200 personnes (d'après les journaux), parmi lesquelles des enfants, auraient reçu des doses plus ou moins importantes. D'autres billes suivirent le destin du métal dans des fonderies fabriquant des barres-armatures pour béton et des pieds tubulaires de table de restaurant. Ces produits contaminés de façon non homogène ont été commercialisés au Mexique et aux Etats-Unis. C'est fortuitement que ce matériel contenant du cobalt 60 fut découvert aux Etats-Unis en février 1984.

\section{LES ÉVÉNEMENTS ACCIDENTELS DE PORTÉE RESTREINTE}

Ce sont ceux qui, par leur nature même, ne peuvent concerner qu'un nombre limité de personnes, le plus souvent atteintes au cours d'activités professionnelles.

On est tout naturellement amené à les classer en deux catégories selon qu'ils ont entraîné des expositions à des rayonnements issus de sources externes ou des expositions à des rayonnements issus de sources qui ont pénétré dans l'organisme.

\section{Les expositions externes accidentelles}

a) Les accidents de criticité

Ce sont des accidents spécifiques de l'énergie nucléaire. Ils peuvent survenir lors de travaux de recherche sur des ensembles sous-critiques expérimentaux d'éléments d'engins explosifs ou de réacteurs de puissance très faible ou nulle, et également au cours d'opérations chimiques impliquant des matières fissiles. Les expositions qu'ils peuvent entraîner concernent les personnes assez proches du système qui diverge, et sont essentiellement des expositions aux neutrons et aux rayonnements $\gamma$. Ces rayonnements sont les uns et les autres pénétrants, et il en résulte une exposition globale (plus ou moins hétérogène) de l'organisme.

De 1945 à 1965 , on a compté dans le monde ${ }^{(1)} 12$ accidents de criticité ayant fait des victimes, au sens donné à ce mot plus haut : 9 aux EtatsUnis, 1 en URSS, 1 en Yougoslavie et 1 en Belgique. Les progrès réalisés dans la maîtrise de la criticité et la mise en place de moyens efficaces

(1) Avec les réserves qui s'imposent pour tenir compte du peu d'informations qui sont publiées dans certains pays. 
de protection du personnel, lorsque cela était possible, ont permis d'éliminer toute exposition due à de tels accidents de 1965 à 1983, année où s'est produit le 23 septembre un grave accident sur un réacteur expérimental en Argentine, qui a entraîné le décès du manipulateur. La transgression des règles les plus élémentaires de sûreté est à l'origine de cette divergence.

De 1945 à 1984, le bilan [8] fait apparaître :

- 9 décès, dont 3 immédiats par effet mécanique explosif ; le temps qui s'écoule entre l'exposition et le décès varie de trente-cinq heures à trentedeux jours ;

- 37 survivants ayant reçu des doses parfois élevées, mais avec des rapports gamma-neutron très variables et des hétérogénéités souvent très marquées dans la distribution spatiale.

\section{b) Les expositions globales aux rayonnements $\gamma$ ou $X$}

Durant la première moitié du $X X^{e}$ siècle, les générateurs de rayons $X$ et les éléments radioactifs naturels furent les principales sources importantes de photons. Avec la découverte de la radioactivité artificielle, le développement des réacteurs pour fabriquer des sources isotopiques et la construction de grands accélérateurs de particules permettant d'obtenir des rayons $X$ de grande énergie, les sources se sont considérablement diversifiées et multipliées.

Les accidents qui ont entraîné de graves expositions globales de l'organisme, souvent accompagnées d'expositions localisées à très fortes doses, sont au nombre de 24 (2 en France). Ils ont été dans leur grande majorité provoqués par des sources isotopiques : iridium 192 (11 accidents sur 24), cobalt 60 (9 sur 24). Les circonstances les plus fréquentes sont de deux sortes : source perdue, ramassée, mise dans la poche ou ramenée à la maison (c'est la séquence classique avec les sources d'appareils de gammagraphie industrielle); et entrée dans les salles d'irradiation par suite de défaillance des systèmes de sécurité, inadvertance ou transgression des consignes.

Le dernier accident en date s'est déroulé au Maroc en juin 1984 ; on a découvert qu'une source d'iridium 192 avait été apportée chez lui par un ouvrier plusieurs semaines auparavant. Les deux parents et quatre enfants sont morts. Trois autres personnes ont été traitées au Centre international de radiopathologie à Paris.

L'ensemble de ces 24 événements accidentels a provoqué 17 décès. Les irradiés survivants sont au nombre de 38 ( 3 en France) parmi lesquels beaucoup ont, en outre, présenté des atteintes localisées justifiant une intervention chirurgicale.

\section{c) Les expositions localisées}

Ce sont les accidents les plus fréquents. De 1945 à 1984, on en a dénombré environ 115 (31 en France) qui ont entraîné des brûlures graves se situant, dans la plupart des cas, au niveau des doigts et des mains.

L'emploi des rayons $X$ dans les travaux de recherche en physique et dans l'analyse par fluorescence est la pratique de loin la plus dangereuse ; elle est suivie de la manipulation en toute inconscience des sources d'iridium 192 principalement utilisées en gammagraphie industrielle.

Le tableau I donne une ventilation des 156 cas (dont 38 en France) d'expositions localisées graves, qui ont pu être recensés entre 1945 et 1984 ; parmi eux, 35 ont fait l'objet d'une intervention chirurgicale. 
TABLEAU I

Expositions localisées graves (1945-1984)

\begin{tabular}{|l|rr|}
\hline Rayons $X$ & 69 & (20 en France) \\
Iridium 192 & 39 & $(7$ en France $)$ \\
Cobalt 60 & 25 & $(4$ en France $)$ \\
Emetteurs $\beta$ & 11 & \\
Accélérateurs & 8 & (4 en France) \\
Sources diverses & 4 & \\
\hline Total & 156 & \\
\hline
\end{tabular}

\section{Les expositions internes}

Les accidents au cours desquels des activités importantes d'éléments radioactifs ont pu être incorporées sont beaucoup plus rares que ceux qui ont entraîné des expositions externes. Cependant, il faut noter que, les critères qui permettraient de classer une exposition interne dans les catégories " graves " ou " pas graves " étant difficiles à déterminer avec précision, les recensements faits à ce jour peuvent présenter des lacunes.

Une liste établie [5] à partir du Registre général tenu à Oak Ridge (DOE - REAC/TS) fait état d'une vingtaine d'accidents ayant entraîné 33 cas qualifiés de "sévères " parmi lesquels on compterait 4 décès : 2 en Allemagne (importante incorporation de tritium), 2 aux Etats-Unis (surdosage au cours d'un traitement avec de l'or 198).

D'une façon générale, les radionucléides les plus souvent rencontrés dans ces accidents sont des plutonium et américium. II faut aussi mentionner un accident de surdosage en yttrium 90, qui se serait produit à Houston en 1980 et qui aurait provoqué la mort de 5 malades.

\section{BILAN GÉNÉRAL}

L'ensemble des données actuellement collectées est présenté dans le tableau II.

Ce bilan qui couvre jusqu'à ce jour toute la période de l'ère dite "nucléaire ", c'est-à-dire près de quarante ans, bien qu'évidemment trop lourd en soi, paraît relativement léger lorsqu'on le compare aux bilans analogues que l'on peut dresser pour les autres activités techniques et industrielles et, en particulier, pour la production d'énergie à partir des sources les plus classiques comme le charbon et le pétrole.

TABLEAU II

Expositions accidentelles (1945-1984)

\begin{tabular}{|c|ccc|}
\hline $\begin{array}{l}\text { Evénements accidentels de } \\
\text { grande portée } \\
\text { Décès } \\
\text { Survivants }\end{array}$ & 2 & 1 & 113 \\
\hline $\begin{array}{l}\text { Evénements accidentels de } \\
\text { portée restreinte } \\
\text { Décés } \\
\text { Survivants }\end{array}$ & 185 & 35 & 263 \\
\hline Total & & 36 & 376 \\
\hline
\end{tabular}




\section{RÉFÉRENCES BIBLIOGRAPHIQUES}

[1] BECQUEREL H., CURIE P. Action physiologique des rayons du radium. C.R. Acad. Sci. 1901, 132, 1289.

[2] CONARD R.A. The 1954 Bikini Atoll incident : an update of the findings in the Marshallese people. In : The medical basis for radiation accident preparedness. (Hubner K., Fry S., Eds.) Amsterdam : Elsevier, 1980,55-58.

[3] DANIEL J. Depilatory action of the X-rays. Med. Record 1986, 49, 595.

[4] DOUSSET M., JAMMET H. Les accidents humains d'irradiation d'origine nucléaire. In : Les irradiations thérapeutiques et accidentelles, Créteil, 6-8 juin 1984. Paris: Masson, 1984,27-61.

[5] FRY S.A. DOE REAC/TS, Oak Ridge. Communication personnelle, 1984.

[6] KUMATORI T., ISHIHARA T., HIRASHIMA K., SUGIYAMA H., ISHLI S., MLYOSHI K. Follow-up studies over a 25-year period on the Japanese fishermen exposed to radioactive fallout in 1954. In: The medical basis for radiation accident preparedness, (Hubner K., Fry S., Eds.) Amsterdam : Elsevier, 1980, 33-54.

[7] MEDVEDEV Z. Two decades of dissidence. New Scientist 1976, 72, 264.

[8] MEDVEDEV Z. Acts behind the Soviet nuclear disaster. New Scientist 1977, 74, 761.

[9] SORAN D.M., STILLMAN D.B. An analysis of the alleged Kyshtym disaster. LA 9217-MS, 1982.

[10] STEVENS L.G. Injurious effects on the skin. Br. Med. J., 1986, 1, 998. 